\title{
DESIGN, ANALYSIS OF 4X4 AND 8X8 MIMO SYSTEM WITH ZF, MMSE AND BF TECHNIQUES
}

\author{
ARUN KUMAR ${ }^{1}$, PIYUSH VARDHAN ${ }^{2}$, MANISHA GUPTA ${ }^{3}$ \\ ${ }^{1}$ Asst.Professor, Dept. of ECE, JECRC University, ${ }^{2}$ Dept. of ECE, JECRC University, ${ }^{3}$ Senior IEEE Member, Dept. of \\ Physics \\ E-mail: arun.kumar@jecrcu.edu.in_Piyushvrdhatech@gmail.com,manisha.gupta@jecrcu.edu.in
}

\begin{abstract}
This work focuses on studying signal detection using three different equalization techniques, namely: Zero Forcing (ZF), Minimum Mean Square Error (MMSE) and Beam Forming (BF), for a 4x4 MIMO-system. Results show that ZF equalization is the simplest technique for signal detection, However, Beam Forming (BF) gives better Bit Error Rate (BER) performances at high Signal to Noise Ratio (SNR) values with some complexity in design. For more antennas at the base station, it is too complex to design the weight matrix for $\mathrm{ZF}$, however, it is suitable for BF with the help of good quality digital signal processors. Performance of MIMOsystem, with 8 antennas at the base station using BF equalization, is analysed to get BER values at different SNR. Results show a considerable improvement in BER for 8 antennas at the base station.
\end{abstract}

Keywords: MIMO system, ZF, MMSE, BF, BER, SNR

\section{INTRODUCTION}

Massive - MIMO systems can send data at a higher speed than conventional MIMO systems are capable of, using spatial multiplexing techniques. At the receiver end, a large number of signals are received in addition to along with the signal from the desired transmit antenna, which act as an interference, thus making signal detection a crucial task for massive - MIMO systems [1]. There are various detection techniques which can be used for this purpose, namely: Zero Forcing (ZF), Minimum Mean Square Error (MMSE) detection and Beam Forming (BF). In a conventional communication system, there is only one antenna at both transmitter and receiver ends (also known as Single Input and Single Output (SISO)) due to which a lot of traffic occurs, which in-turn reduces the capacity of the system. The current situation needs huge capacity due to the increasing numbers of subscribers [2]. A comparative study of different linear pre-coding schemes like BF and Regularized Zero Forcing (RZF) for TDD downlink Massive-MIMO gives results for path loss, channel estimation, pilot contamination and arbitrary antenna correlation which suggest that RZF, which is relatively a simple pre-coding scheme, gives the same performance with a much smaller number of antennas per user terminal (UT) as compared to the BF which is complex to calculate [3]. An energy efficient MassiveMIMO system can be designed for high SNR values using interference-suppressing pre-coding schemes like ZeroForcing (ZF) pre-coding [4, 5]. Zero-Forcing (ZF) equalization is in practice very close to the channel capacity of downlink and uplink of a Massive- MIMO for pre-coding and detection. The inverse of the KxK matrix (which gives the weight matrix for ZF equalization) is very complex to calculate and implement for the hardware design of a massive-MIMO system with large number of antennas at the base station. For a large number of base station antennas (say M) and comparatively less number of users (say $\mathrm{K}$, where $\mathrm{M}>>\mathrm{K}$ ), $\mathrm{M} / \mathrm{K}$ ratio is derived using suitable matrix inversion approximation (Neuman Series (NS) for Matrix Inversion Approximation (MIA)). Using NS-based MIA, calculation of inverse of KxK matrix gets simplified, making it suitable for hardware implementation [6 - 8].

\section{SYSTEM MODEL}

Let us consider a $\mathrm{N}_{\mathrm{R}} \times \mathrm{N}_{\mathrm{T}}$ massive-MIMO system as shown in the figure (1), where $N_{R}$ is the number of receiver antennas and $\mathrm{N}_{\mathrm{T}}$ is the number of antennas at the transmitter. Let us consider that all the antennas are uncorrelated.

\subsection{Mathematical Model}

Let $\bar{H}$ denotes a channel matrix and $\mathrm{h}_{\mathrm{ji}}$ denotes the channel gain between the ith transmit antenna and the $\mathrm{jth}$ receive antenna, where $\mathrm{j}=1,2,3 \ldots \ldots \mathrm{N}_{\mathrm{R}}$ and $\mathrm{i}=1,2,3 \ldots \ldots \ldots$ $\mathrm{N}_{\mathrm{T}}$. Let the multiplexed transmit data signal is denoted by $\bar{X}=\left[\mathrm{x}_{1}, \mathrm{x}_{2}, \ldots \ldots, \mathrm{x}_{\mathrm{N}_{\mathrm{T}}}\right]^{\mathrm{T}}$ where $x_{i}$ denotes the transmit signal from the $i$ th transmit antenna. The Received data signal is denoted by $\bar{Y}=\left[y_{1}, y_{2}, \ldots \ldots, y_{N_{R}}\right]^{\mathrm{T}}$ where $y_{j}$ denotes the received signal at the jth receive antenna. Let $n_{j}$ denotes the white Gaussian noise, at the jth receive antenna, with a variance $\sigma_{n}^{2}$.

The $\mathrm{N}_{\mathrm{R}} \times \mathrm{N}_{\mathrm{T}}$ massive-MIMO system can be represented mathematically as

$$
\begin{gathered}
\bar{Y}=\bar{H} \bar{X}+\bar{n} \\
\bar{Y}=\overline{\mathrm{h}}_{1} \mathrm{x}_{1}+\overline{\mathrm{h}}_{2} \mathrm{x}_{2}+\cdots+\overline{\mathrm{h}}_{\mathrm{N}_{\mathrm{T}}} \mathrm{x}_{\mathrm{N}_{\mathrm{T}}}+\overline{\mathrm{n}}
\end{gathered}
$$

Where $\bar{n}=\left[n_{1}, n_{2}, \ldots \ldots, n_{N_{R}}\right]^{\mathrm{T}}$ and $\bar{h}_{i}$ denotes the $i$ th column vector of the channel matrix $\overline{\mathrm{H}}$.

In matrix form MIMO system model can be written as, 


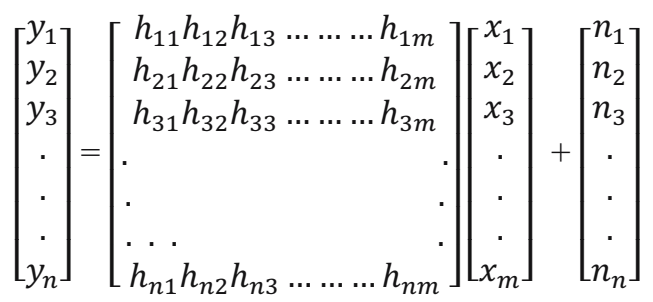

Here $h_{i j}=\alpha+j \beta$ (complex channel).

For a sufficiently rich scattering environment with no line of sight (LOS), the channel is a Raleigh Channel with channel gain $\left(\left|h_{i j}\right|\right)$ where $\alpha$ and $\beta$ are normally distributed independent random variables. Noise power for each noise component at each receiver antenna is given as

$$
\mathrm{E}\left(\left|n_{i}\right|^{2}\right)=\sigma_{n}^{2}
$$

The covariance matrix for noise is given mathematically as

$$
R_{n}=\mathrm{E}\left(\bar{n} \bar{n}^{H}\right)=\sigma_{n}^{2} \mathrm{I}
$$

The noise at each antenna is uncorrelated and white across the space, hence known as spatial noise.

\subsection{Signal Detection}

For the above mentioned model of massive - MIMO we use here two kinds of linear signal detectors, namely ZF and MMSE and an angle based signal detector named as $\mathrm{BF}$. To detect the desired signal from the target transmit antenna at receiving end, all the interference signals are nullified or minimized by inverting the effect of the channel by multiplying a suitable weight matrix $\bar{W}$, such that

$$
\widetilde{\mathrm{X}}=\left[\widetilde{\mathrm{x}}_{1}, \widetilde{\mathrm{x}}_{2}, \ldots, \widetilde{\mathrm{x}}_{\mathrm{N}_{\mathrm{T}}}\right]^{\mathrm{T}}=\overline{\mathrm{W}} \overline{\mathrm{Y}}
$$

i.e. the each detected symbol is represented by the linear combination of receiving signals.

\section{A. Zero Forcing Signal Detection}

In Zero forcing signal detection the interferences are nullified by weight matrix $\mathrm{W}_{\mathrm{ZF}}$, where

$$
\mathrm{W}_{\mathrm{ZF}}=\left(\mathrm{H}^{\mathrm{H}} \mathrm{H}\right)^{-1} \mathrm{H}^{\mathrm{H}}
$$

It inverts the effect of the channel as

$$
\begin{gathered}
\overline{\mathrm{X}}_{\mathrm{ZF}}=\mathrm{W}_{\mathrm{ZF}} \overline{\mathrm{Y}} \\
\overline{\mathrm{X}}_{\mathrm{ZF}}=\overline{\mathrm{X}}+\left(\mathrm{H}^{\mathrm{H}} \mathrm{H}\right)^{-1} \mathrm{H}^{\mathrm{H}} \cdot \bar{n} \\
\overline{\mathrm{X}}_{\mathrm{ZF}}=\overline{\mathrm{X}}+\overline{\mathrm{n}}_{\mathrm{ZF}}
\end{gathered}
$$

Where, $\overline{\mathrm{n}}_{\mathrm{ZF}}=\mathrm{W}_{\mathrm{ZF}} \overline{\mathrm{n}}=\left(\mathrm{H}^{\mathrm{H}} \mathrm{H}\right)^{-1} \mathrm{H}^{\mathrm{H}} \cdot \bar{n}$

Noise power can be calculated as $\left\|\bar{n}_{Z F}\right\|_{2}^{2}$.

The expected value of noise power is given as (Figure 1)

$$
\mathrm{E}\left\{\left\|\bar{n}_{Z F}\right\|_{2 .}^{2}\right\}=\sum_{i=1}^{N_{r}} \frac{\sigma_{n}^{2}}{\sigma_{i}^{2}}
$$

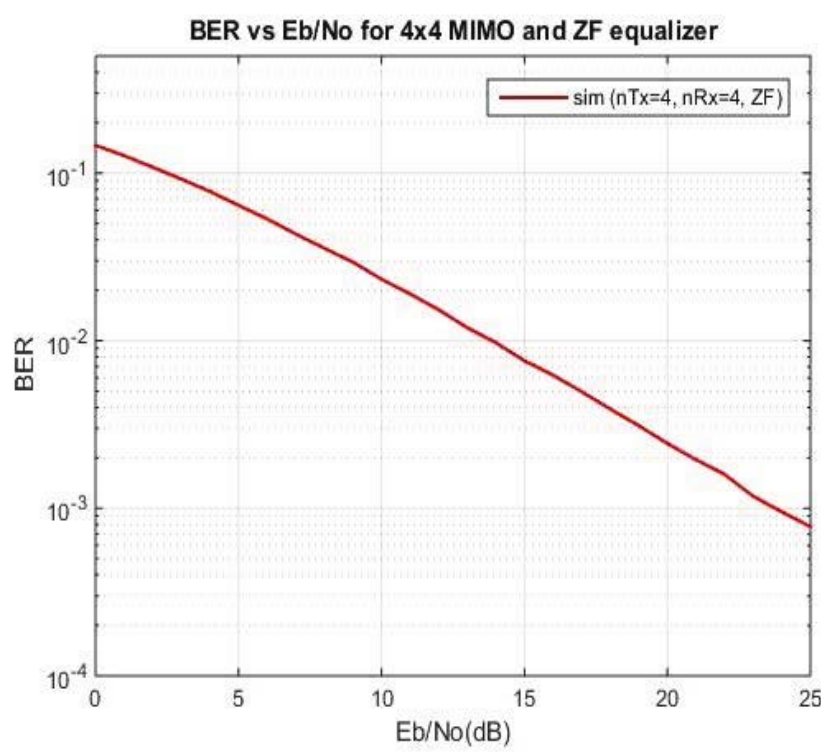

Figure 1. Simulation result BER vs Eb/No for $4 \times 4$ MIMO using $\mathrm{ZF}$ equalizer

\subsection{MMSE Signal Detection}

In MMSE signal detection the interference is minimized by enhancing the post detection signal-to-interference plus noise ratio (SINR) by multiplying the received signal with MMSE weight matrix, given as:

$$
\mathrm{W}_{\text {MMSE }}=\left(\mathrm{H}^{\mathrm{H}} \mathrm{H}+\sigma_{n}^{2} \mathrm{I}\right)^{-1} \mathrm{H}^{\mathrm{H}}
$$

It minimizes the interference by inverting the channel effect as

$$
\begin{gathered}
\overline{\mathrm{X}}_{\text {MMSE }}=\mathrm{W}_{\text {MMSE }} \overline{\mathrm{Y}} \\
\overline{\mathrm{X}}_{\text {MMSE }}=\left(\mathrm{H}^{\mathrm{H}} \mathrm{H}+\sigma_{\mathrm{n}}^{2} \mathrm{I}\right)^{-1} \mathrm{H}^{\mathrm{H}} \overline{\mathrm{Y}} \\
\overline{\mathrm{X}}_{\text {MMSE }}=\overline{\mathrm{X}}+\left(\mathrm{H}^{\mathrm{H}} \mathrm{H}+\sigma_{\mathrm{n}}^{2} \mathrm{I}\right)^{-1} \mathrm{H}^{\mathrm{H}} \overline{\mathrm{n}} \\
\overline{\mathrm{X}}_{\text {MMSE }}=\overline{\mathrm{X}}+\bar{n}_{M M S E}
\end{gathered}
$$

Where, $\bar{n}_{M M S E}=\left(\mathrm{H}^{\mathrm{H}} \mathrm{H}+\sigma_{\mathrm{n}}^{2} \mathrm{I}\right)^{-1} \mathrm{H}^{\mathrm{H}} \overline{\mathrm{n}}$

The MMSE receiver requires the statistical information about the noise $\left(\sigma_{\mathrm{n}}^{2}\right)$.

Noise power can be calculated as $\left\|\bar{n}_{M M S E}\right\|_{2}^{2}$.

The expected value of noise power is given as

$$
\mathrm{E}\left\{\left\|\bar{n}_{M M S E}\right\|_{2}^{2}\right\}=\sum_{i=1}^{N_{T}} \frac{\sigma_{n}^{2} \cdot \sigma_{i}^{2}}{\left(\sigma_{i}^{2}+\sigma_{n}^{2}\right)^{2}}
$$

For a $4 \times 4$ MIMO system, simulation results for BER using MMSE equalization is given in Figure 2. 


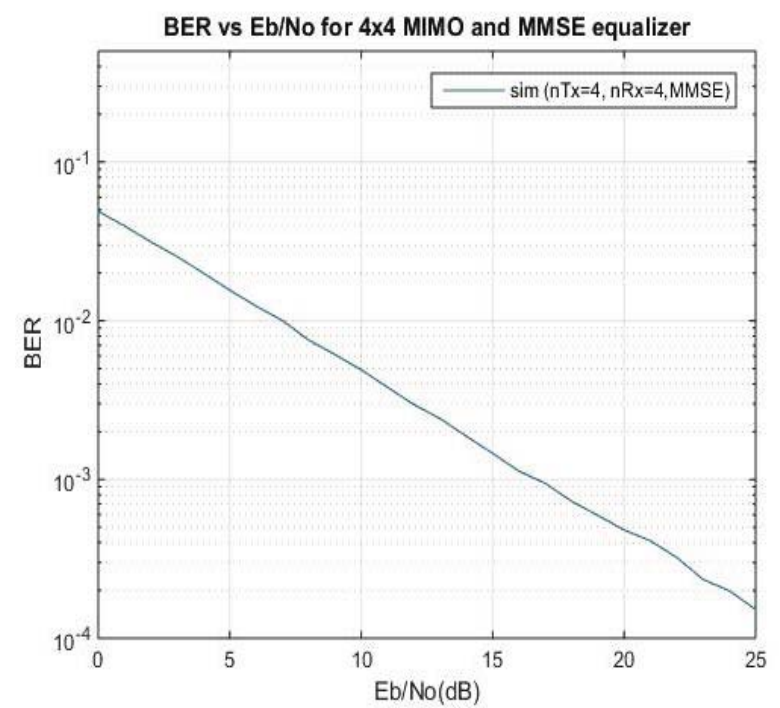

Figure 2. Simulation result BER vs Eb/No for $4 \times 4$ MIMO using MMSE equalizer

\section{BEAM FORMING}

In beam forming signal detection technique we use closeloop transmit diversity scheme where the channel is already known to the receiver. To minimize the effect of the channel, the symbol transmitted from each transmit antenna is multiplied by a complex number which is equal to the inverse of the phase of the channel, so that all the received signals at a receiver leads to constructive interference.

Mathematically, received signal (without BF) is given as:

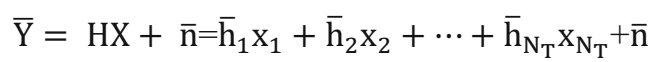

In matrix form

$$
\mathrm{y}=\left[\begin{array}{ll}
h_{1} h_{2} h_{3} \ldots \ldots \ldots h_{N_{T}}
\end{array}\right]\left[\begin{array}{c}
x_{1} \\
x_{2} \\
x_{3} \\
\cdot \\
\cdot \\
\cdot \\
x_{N_{T}}
\end{array}\right]+\mathrm{n}
$$

Received signal (with BF)

$$
\mathrm{y}=\left[\begin{array}{lll}
h_{1} h_{2} h_{3} & \ldots \ldots & \ldots . . h_{N_{T}}
\end{array}\right]\left[\begin{array}{c}
e^{-j \theta 1} \\
e^{-j \theta 2} \\
e^{-j \theta 3} \\
\cdot \\
\cdot \\
\cdot \\
e^{-j \theta N_{T}}
\end{array}\right]\left[\begin{array}{c}
x_{1} \\
x_{2} \\
x_{3} \\
\cdot \\
\cdot \\
\cdot \\
x_{N_{T}}
\end{array}\right]+\mathrm{n}
$$

Where, hi $=\left|h_{i}\right| e^{j \theta i}$

Thus, the signal received at the receiver is

$$
\mathrm{y}=\left(\left|h_{1}\right|+\left|h_{2}\right|+\cdots+\left|h_{N_{T}}\right|\right) \mathrm{x}+\mathrm{n}
$$

To invert the effect of the channel, the received symbols are multiply by the inverse of the channel matrix obtained using beam forming. The detected symbol can be expressed mathematically as

$$
\tilde{y}=\frac{y}{\left(\left|h_{1}\right|+\left|h_{2}\right|+\cdots+\left|h_{N_{T}}\right|\right)}=\mathrm{x}+\frac{n}{\left(\left|h_{1}\right|+\left|h_{2}\right|+\cdots+\left|h_{N_{T}}\right|\right)}
$$

For a 4x1 MIMO system, simulation results for BER using $\mathrm{BF}$ equalization is given below (Figure 3 ).

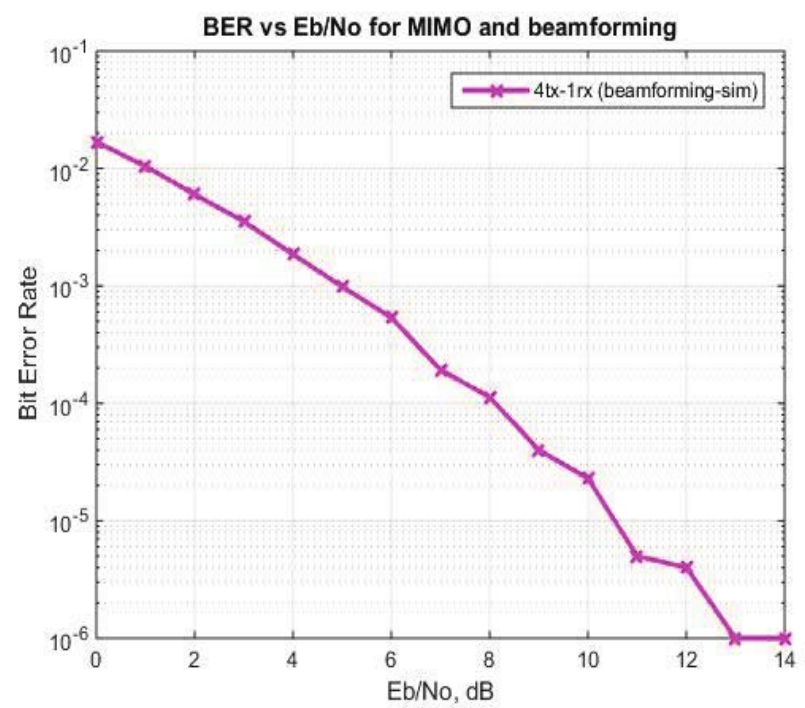

Figure 3. Simulation result BER vs Eb/No for 4x1 MIMO using $\mathrm{BF}$ equalizer

For an 8x1 MIMO system, simulation results for BER using BF equalization is given below (Figure 4).

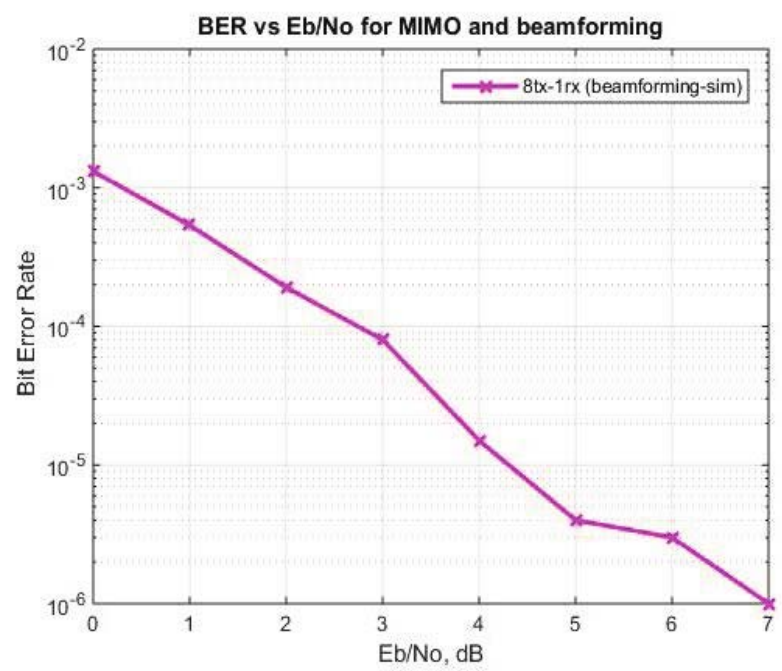

Figure 4. Simulation result BER vs Eb/No for 8x1 MIMO using BF equalizer

\section{RESULTS AND DISCUSSION}

Simulation results for $4 \times 4$ MIMO-Systems, using three different equalization techniques (ZF, MMSE and Beam Forming), are analysed to obtain the behaviour of their performances based on BER. Zero forcing equalization is trying to nullify the interference and gives a moderate 
BER value for higher SNR as it is easy to implement practical due to less complexity. The simulation results show that the MMSE is comparatively better technique for post detection equalization. Tabular values show that MMSE gives least BER for moderate SNR values. However, it is comparatively complex to design and implement practically. Another technique called Beam Forming is used here to minimize the interferences and enhance system performance. Simulation results show tremendously positive results for Beam Forming. For higher SNR, almost zero BER is observed for large amounts of transmitting bits. Simulation results for the 8x1 MIMO show that BER is considerably reduced even at lower SNR if we use beam forming.

\section{CONCLUSION}

In this work we have tried to implement the MassiveMIMO systems with its mathematical model and related simulation results. A 4x4 basic model of MIMO systems is designed using different equalization techniques for post detection namely, ZF, MMSE and Beam Forming. Simulation results show that although $\mathrm{ZF}$ equalization is simplest to implement for a $4 \times 4$ system, but it is much more complex for more antenna at base station. Based on the simulation results it is clear that beam forming $(\mathrm{BF})$ is the best suitable method for massive-MIMO systems (that is, for a system with large number of antenna at the base station). A further improved system with better SNR and less BER using Beam Forming, with 8 antennas at the base station (8x1 MIMO System) is simulated. Simulation results obtained are robust and favourable with less BER. For next generation cellular communication systems, Beam Forming is one of the best suitable techniques for signal detection and pre-coding. In practice, a lot of limitations arise due to the correlation of antennas and hardware impairments.

\section{REFERENCES}

[1] Daniel W. Bliss, Keith W. Forsythe, M. Amanda, MIMO wireless communication, Lincoln Lab, 15 (1), pp. 97-126, 2005.

[2] T. Yasodha., Decoding for $8 * 8$ MIMO System using Convolution Coding implemented in VLSI, Int J Eng Res Appl, 3 (1), pp. 278-282, 2013.

[3] J. Hoydis, C. Hoek, T.Wild, Stephen Brink., Channel measurements for large antenna arrays, Proc. Int. Symp. Wireless Communication. Syst. (ISWCS), pp. 811-815, 2012.

[4] Bjornson, E., Hoydis, J., Kountouris, M., Debbah, M., Massive MIMO systems with non-ideal hardware: Energy efficiency, estimation, and capacity limits, IEEE Transactions on Information Theory, 60(11), pp.7112-7139, 2014.

[5] K. Sengar, N. Rani, A. Singhal, D. Sharma, S. Verma, T. Singh., Study and capacity evaluation of SISO, MISO and MIMO RF wireless communication systems, Int J Eng Trends Technology (IJETT), 9 (9), pp. 437-440, 2014.

[6] Zhu, D., Li, B. \& Liang, P., On the Matrix Inversion Approximation Based on Neumann Series in
Massive-MIMO systems, IEEE ICC- Wireless Communication Symposium, pp .1763-1769, 2015.

[7] Kumar A., Gupta M., Design, Development of MC-CDMA, and Reduction of ISI for Different Modulation Techniques, pp.81-91, 2014.

[8] Kumar A., Gupta M., Design, comparative study and analysis of CDMA for different modulation techniques, Egyptian Informatics Journal, 16(3), pp. 351-365, 2015 